\title{
Chapter 4: Derivations and Observations of Prominence Bulk Motions and Mass 1
}

\author{
T.A. Kucera \\ NASA Goddard Space Flight Center, Code 671, Greenbelt, MD 20771, USA
}

\begin{abstract}
In this chapter we review observations and techniques for measuring both bulk flows in prominences and prominence mass. Measuring these quantities is essential to development and testing of models discussed throughout this book. Prominence flows are complex and various, ranging from the relatively linear flows along prominence spines to the complex, turbulent patterns exhibited by hedgerow prominences. Techniques for measuring flows include time slice and optical flow techniques used for motions in the plane of the sky and the use of spectral line profiles to determine Doppler velocities along the line of sight. Prominence mass measurement is chiefly done via continuum absorption measurements, but mass has also been estimated using cloud modeling and white light measurements.
\end{abstract}

\section{Bulk Motions}

Prominences have long been known to be dynamic structures, displaying internal motions of various kinds even when globally at rest. Here we discuss bulk motions and methods used for measuring them, although the measurement methods are also relevant to the oscillatory motions discussed in Chapter 11 (Ballester 2015).

A good understanding of flows is highly important for testing and constraining models of prominence formation and stability, determining the role of flows in the force and energy equilibria of prominences, and casting light on closely related questions concerning prominence magnetic field structure. For instance, evaporative-condensation models predict that flows of cool material should originate in the corona, while injection and levitation models involve cool material flowing upwards form the chromosphere. If we assume that flows are moving along magnetic field lines then the flow trajectories can tell us about the magnetic structure of the prominence, but it could also be the case that the material is moving with a changing field or even in some cases diffusing across the field. The various theoretical models of prominence magnetic field and related dynamics and their predictions

\footnotetext{
${ }^{1}$ In Solar Prominences, eds. J.-C. Vial \& O. Engvold, Springer (2015)
} 
are discussed in more detail in Chapter 10 (Karpen 2015).

To obtain the required information we need detailed trajectories of prominence plasma features, including the origin of the plasma, and any changes in temperature and velocity. How do observed motions compare to those expected from various models of the prominence magnetic field and actual magnetic field measurements? What connections, if any, are there between flows at different temperatures? What processes can explain these observed flows?

In the last few decades, new instrumentation has yielded more information concerning flows. In the visible regime, the new high temporal and spatial resolution data combined with spectral information have revealed counter-streaming flows in filaments on the disk in observations such as those from the the Swedish Solar Telescope (SST) at La Palma. The similarly good spatial resolution of the Hinode/Solar Optical Telescope (SOT) combined with excellent long term image stability allowed by its space based platform have allowed detailed studies of the complex motions observed in prominences at the limb.

In the UV and EUV range spectrographs such as Solar and Heliospheric Observatory (SOHO)'s Coronal Diagnostic Spectrometer (CDS) and Solar Ultraviolet Measurements of Emitted Radiation (SUMER) and Hinode's EUV Imaging Spectrometer (EIS) have provided information in a range of lines formed at chromospheric, transition region, and coronal temperatures. The new Interface Region Imaging Spectrograph (IRIS) mission provides higher resolution spectroscopic as well as plane-of-sky information concerning the motions of plasma at chromospheric and transition region temperatures. High cadence UV and EUV imaging information from the Solar Dynamics Observatory (SDO)'s Atmospheric Imaging Assembly (AIA) and, earlier, from the Transition Region and Coronal Explorer (TRACE) and $\mathrm{SOHO} /$ Extreme ultraviolet Imaging Telescope (EIT) have been important as well.

\subsection{Measurements of Motions}

Motions in the plane of the sky are measured by tracking actual features, while line of sight (LOS) motions are detected using Doppler shifts. Sometimes the plane-of-sky (POS) and line position methods can be combined to good effect. With either LOS or POS measurements alone, we have no direct information on the 3D structure. However, in some cases we can make estimates based on the knowledge of the orientation of prominence features as viewed over several days or from two points of view using the Solar Terrestrial Relations Observatory (STEREO) spacecraft. 


\subsubsection{Motions in the Plane of the Sky}

Observations of motions in the plane of the sky have the advantage that it is possible to pick out actual moving features, making it straight forward in some cases to track the motion by simply marking off the feature location in successive images.

There are a number of general things to be careful of, however. The very fact that such measurements are feature driven makes it possible to miss evenly moving flows that vary below the resolution of the data. Also, there is the question of what is actually moving. Often a variation in intensity may indeed be due to actual moving material, but it is also possible it is due to a propagating disturbance in density or temperature. There are cases, especially in optically thin plasmas, in which multiple layers of plasma can produce confusing intensity variations and make the data hard to interpret. It is important to have sufficiently high spatial and temporal resolution to avoid aliasing and be able to reliably identify and track particular features. One should also consider projection effects. For instance, when a prominence is seen along the limb it can be difficult to distinguish vertical motions from horizontal flows curving over the solar limb.

Time-Slice A common variation of feature tracking is to use the time slice method (see Fig. 1). The intensity or velocity along a trajectory traced out in consecutive images of the filament is followed versus time, and the slope of the brightening or darkening in this $2 \mathrm{D}$ diagram gives a measurement of the velocity of the feature in the plane of the sky. This method can be easily applied to high spatial resolution images, e.g., with the SST or the Dutch Open Telescope (DOT) at La Palma, and has also been used with EUV images. The slices can be linear, but they can also curve to follow the trajectory of a particular feature.

Optical Flow Techniques There has also been some use of automatic tracking to trace prominence flow fields. This can be difficult because of the complex nature of prominences, often including multiple sources that may be optically thin or combine both emission and absorption. The techniques that have been used successfully are best applied to areas that can be described in terms of flow fields rather than discrete moving features.

One of the most commonly used of these methods is Local Correlation Tracking (LCT) (Leese et al. 1970), in which a two dimensional cross-correlation is applied to a pair of images to determine the shifts for different parts of the images so as to maximize the function (November \& Simon 1988)

$$
C(\delta, x)=\int J_{t}\left(\xi-\frac{\delta}{2}\right) J_{t+\tau}\left(\xi+\frac{\delta}{2}\right) W(x-\xi) \partial \xi
$$

where $J_{t}(x)$ and $J_{t+\tau}(x)$ are the two images taken at times $t$ and $t+\tau$ respectively, with a vector displacement $\delta$ between them. $W(x)$ is a windowing function that controls the size of the region over which the images are compared.

Also used are optical flow techniques originally developed for magnetogram analysis, 

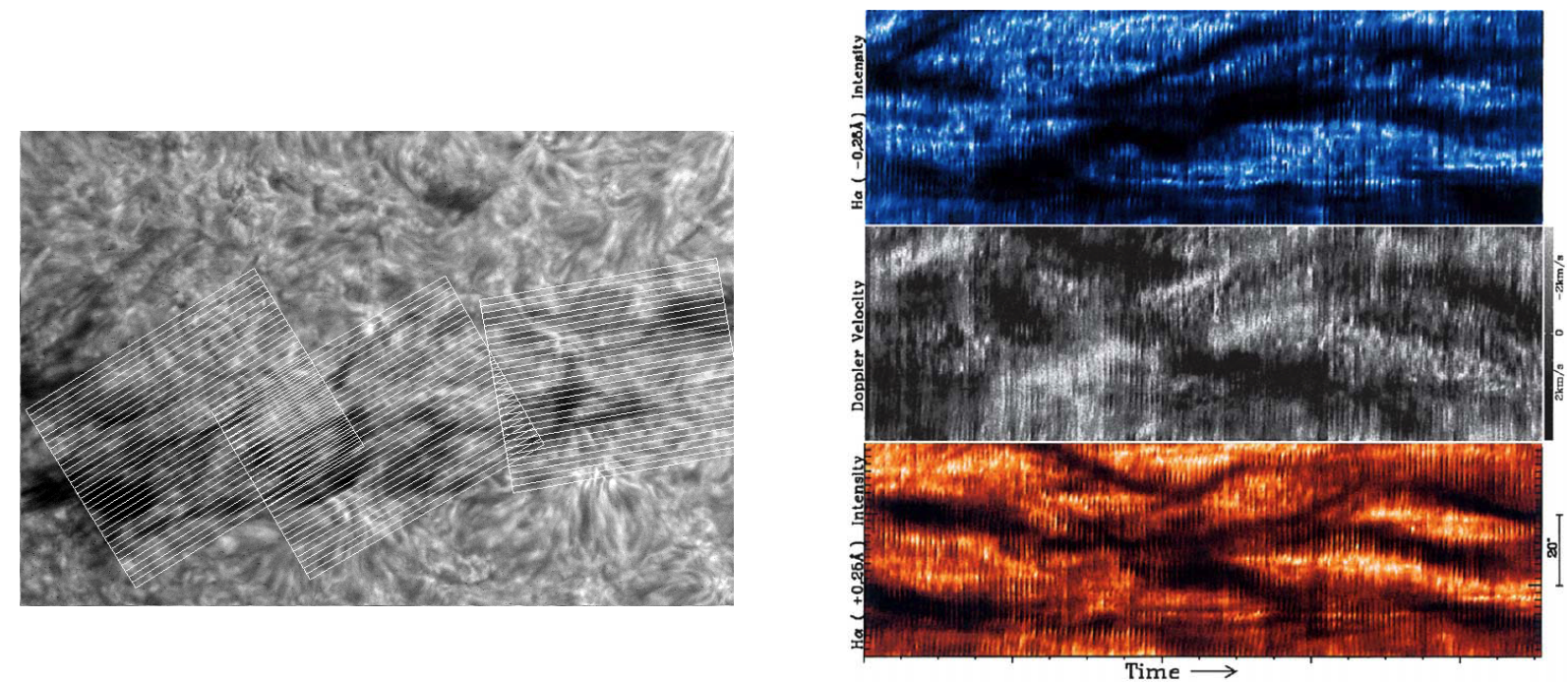

Fig. 1.- An illustration of the use of time slice diagrams to analyze flows in a $\mathrm{H} \alpha$ filament observed with the SST (Lin et al. 2003). The left panel shows the locations of parallel slices used for analyzing velocities in $\mathrm{H} \alpha$ fine structures. At right from top to bottom are the blue wing intensities, Doppler shift, and red wing intensities along a single time slice located in the middle section of the filament. Diagonal features show motions along the slice. 
such as the nonlinear affine velocity estimator (NAVE) (Schuck 2006) which was used to track the flows shown in Fig. 2. These algorithms utilize spatial derivatives of the images and allow for more complex local flows (Chae \& Sakurai 2008).

\subsubsection{Along the Line of Sight: Doppler Observations}

Doppler measurements more reliably give access to steady flows. If there is sufficient spectral resolution the Doppler velocity is generally determined by fitting the observed spectral line, usually with a Gaussian function, and applying the standard formula for Doppler shifts,

$$
v / c=\left(\lambda-\lambda_{0}\right) / \lambda_{0}
$$

where $\lambda_{0}$ is the rest wavelength of the line, $\lambda$ the line wavelength of the source, $v$ the source velocity, and $c$ the speed of light.

When specifying a Doppler velocity it is important to be clear how the zero velocity point is determined. In many cases one cannot assume that the data one is using has been calibrated with respect to the absolute wavelength. The wavelength scale often shifts in the instrument due to instrumental heating or, for ground based instruments, changes in the refractive index of the atmosphere. Although it is possible for some wavebands to incorporate a wavelength standard into an instrument this is not often done in space-based solar spectrographs.

One method of velocity calibration is to select a feature in the field of view or that has been recently observed and declare it to be at rest so that the velocity values are relative to that feature. For spectral observations in visible wavelengths one should optimally precede or follow one's observations with observations of a quiet area at disk center. The average spectral profile over the region is then used to determine the rest wavelength position. The wavelength scale is also sometimes calibrated by measuring it compared to certain spectral lines that are assumed to be at rest. For instance, for UV or EUV observations of the solar disk absolute wavelength calibrations are often done by assuming that chromospheric spectral lines averaged over a large area are at rest. If the Doppler shifts of interest are to be measured using a spectral line produced at chromospheric or transition region temperatures observed above the limb (as is the case for a prominence), a coronal line at a near-by wavelength (again averaged over a large area) might be used as a standard. However, coronal plasma may not be at rest either. Thus one should be aware of the uncertainties in the chosen method and consider how well the absolute Doppler shift has been measured.

A spectrally resolved profile is generally fit with some function, usually a Gaussian. Often there is more than one Doppler component present. This is especially likely in optically thin plasmas, where the emission is an integration of the LOS velocity of many different features so that the measurement provides a value averaged along the LOS. It is 


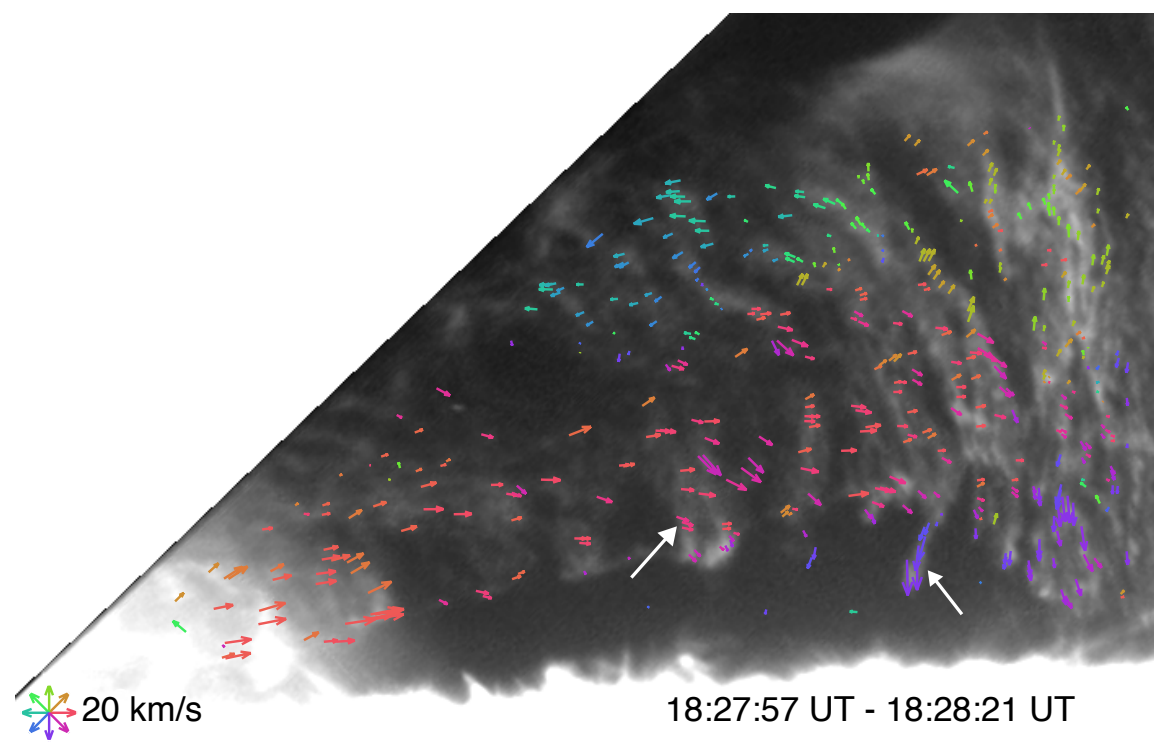

Fig. 2.- Vertically oriented features seen in a hedgerow prominence observed with Hinode/SOT in the $\mathrm{H} \alpha$ line (Chae et al. 2008). They appear to move both horizontally and vertically in the plane of the sky. White arrows point out a newly formed feature and one which is falling downwards.
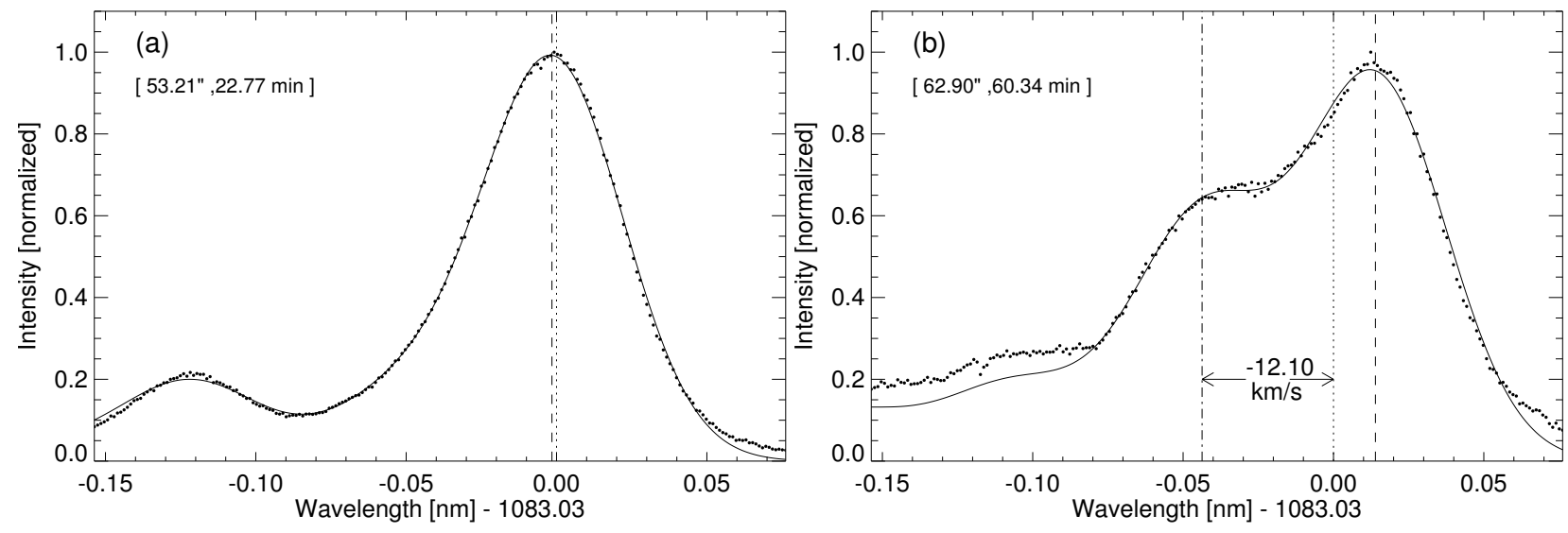

Fig. 3.- Example of a multi-component Doppler shift in a prominence footpoint in He I $10830 \AA$ (Orozco Suárez et al. 2012). (a) shows a rest profile consisting of a weak line at $10829.09 \AA$ (due to the ${ }^{3} S_{1^{-}}{ }^{3} P_{0}$ transition) and the main line at $10830.29 \AA\left({ }^{2} S_{1^{-}}{ }^{3} P_{1}\right.$ and $\left.{ }^{2} S_{1-}{ }^{3} P_{2}\right)$. (b) shows a spectrum with two Doppler components in the main line, one red shifted and one blue shifted. A spectral fit with a single component might indicate little or no motion along the line of sight. The measurements were made with data from Tenerife Infrared Polarimeter attached to the German Vacuum Tower Telescope. 
possible, for instance, for some of the plasma in the field of view to be relatively stationary and for other parts of it to be moving. An example is shown in Fig. 3. Here, fitting the entire spectral line as a unit could result in a measurement of almost no shift at all. At best one would be left with the impression of a broadened line profile with no information concerning the details of the flow. Instead, the line has been fit by two Gaussian functions, indicating a blue shifted component moving at $12 \mathrm{~km} \mathrm{~s}^{-1}$ and a red shifted component at about $4 \mathrm{~km} \mathrm{~s}^{-1}$ (Orozco Suárez et al. 2012).

A related issue is that of line blending, in which multiple lines are present. If all the lines are from the same source they will move in tandem, but if they are not (if, for instance, they are formed at different temperatures in different regions) fitting the line as a single source will again yield incorrect results. Even if two blended lines are formed from the same ion, a density dependence in their ratio can lead to spurious Doppler signals.

Another aspect to Doppler line fitting is that of the reversed lines, which is particularly relevant to prominences. Some optically thick lines exhibit central reversals. Full modeling of the behavior of such lines is discussed in Chapter 5 (Heinzel 2015), but the location of the center of the absorption feature can be an indicator of the relative velocities of different parts of the structure.

For observations of filaments on the disk, the signal coming from the prominence must be disentangled from the chromospheric background. Various techniques have been developed to do this, principally based on cloud model methods (Beckers 1964, Mein et al. 1996; Tziotziou 2007). With such methods, the data is fit to a non-LTE model model of the prominence as a "cloud" suspended above the chromosphere utilizing four parameters: the LOS velocity $V$, the source function $S$, the optical thickness $\tau$, and the line width $\Delta \lambda_{D} . S$ and $\tau$ are strongly coupled but $V$ can be computed. Cloud models are described in more detail in Chapter 5 (Heinzel 2015)

For all these caveats and difficulties, there are short-cuts that are taken. Lines are sometimes sampled at only two or three wavelengths. Differences in the intensities of the red and blue wings of the lines are then used to identify general regions of flow or to separate out material flowing in different directions.

Optimally measurement of flows in and transverse to the plane of the sky can be combined to reveal the three dimensional trajectory of the motion. One particular method used to combine the two is to trace motions using an image slice method applied to the red or blue wing of a spectral line. An example of this is shown in Fig. 1, in which a time slice analysis is done in the red and blue wings of the $\mathrm{H} \alpha$ line, which show different features (Lin et al. 2003). 


\subsection{Observations of Flows in Prominences}

Prominences show a variety of different flows. Here we describe some of the characteristic ones for non-erupting prominences. Eruptions and eruption precursors are discussed in Chapters 15 and 16 (Webb 2015; Gopalswamy 2015).

\subsubsection{Flows in Quiescent Prominences}

What are referred to as quiescent prominences exhibit a range of flow behaviors including motions of vertically aligned structures in hedgerow prominences and also the motions in the spine and barb formations of lower latitude prominences in quiet regions. Although traditionally quiescent prominences are thought of as relatively stationary, even the most stable quiescent filaments are dynamic structures exhibiting flows and oscillations.

Spine flows Quiescent prominences not in the polar crown often show a twopart structure of a long spine accompanied by barbs which extend down to the chromosphere (Engvold 2015). Observations in $\mathrm{H} \alpha$ show flows along prominence spines and barbs at speeds of $10-20 \mathrm{~km} \mathrm{~s}^{-1}$. As shown in the schematic in Fig. 4, the flows can go in both directions simultaneously along the spine, a phenomenon known as counterstreaming (Zirker et al. 1998). These observations report individual moving features traced over distances of 10,000 to $100,000 \mathrm{~km}$. Other observations of spine flows in an intermediate prominence show moving features changing direction, suggesting counter-streaming may be the result of plasma oscillating along the magnetic field (Ahn et al. 2010).

Although polar crown prominences often appear to consist mostly of barbs when observed in $\mathrm{H} \alpha$, observations in the $304 \AA$ EUV imaging band show flowing material along spines, evidently too hot or optically thin to be observed in $\mathrm{H} \alpha$ but apparent in the more optically thick He II $304 \AA$ line.

He II $304 \AA$ emitting plasma has been observed moving along prominence spines at speeds up to $75 \mathrm{~km} \mathrm{~s}^{-1}$ in the plane of the sky (Wang 1999), much faster than considered typical for $\mathrm{H} \alpha$ flows, and similar observations have been made in other transition region temperature lines in the EUV (Kucera et al. 2003).

Flows in Barbs Although barbs in polar crown prominences often take the form of hedgerows (see below), in lower latitude prominences they are narrower structures, appearing as short outgrowths from the spine against the disk in $\mathrm{H} \alpha$ and as thin dark pillars in EUV images of prominences along the limb. These EUV pillars exhibit swaying motions that can be interpreted as material oscillating back and forth (Panasenco et al. 2014), perhaps in dipped field lines. Alternatively, it has been suggested based on Doppler data that these motions may be due to helical motions around a roughly vertical axis (Orozco Suárez et al. 2012). This seems hard to reconcile with the appearance of these structures 


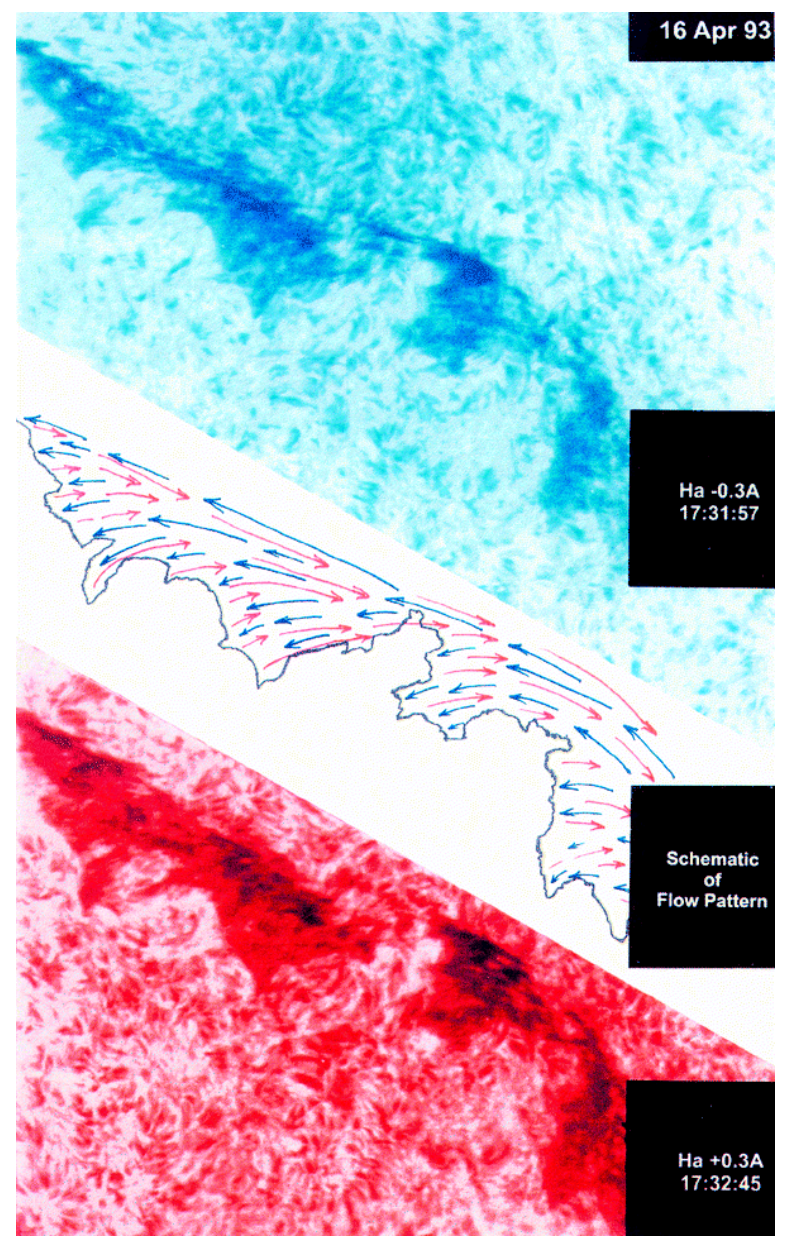

Fig. 4.- Counter-streaming observed in along a prominence spine and barbs observed at Big Bear Solar Observatory (Zirker et al.|1998). The flows were detected by constructing movies from images in the blue wing (top) and red wing (bottom) of the $\mathrm{H} \alpha$ line. 
from above. From that point of view they appear to consist of relatively straight threads.

Hedgerow Prominence Flows Hedgerow prominences (Engvold 2015) tend to form in the polar crown. As mentioned above, hedgerows as seen in $\mathrm{H} \alpha$ are often interpreted as large barbs, with the prominence spine not strongly visible in $\mathrm{H} \alpha$ but observed in $\mathrm{He}$ II 304 Å line.

Hedgerows barbs as seen in $\mathrm{H} \alpha$ often show structures that are aligned perpendicular to the solar limb. These structures have been observed for many years, but recent high resolution observations have renewed focus on these features and related motions.

The Hinode SOT instrument in particular has made possible images of prominences and prominence flows at the limb with unprecedented resolution and stability. Observations of large, quiescent hedgerow prominences in the $\mathrm{Ca}$ II $\mathrm{H}$ and $\mathrm{H} \alpha$ lines show vertically aligned bright and dark features. An example of the bright features is shown in Fig. 2, which shows a series of bright vertical structures exhibiting vortical-type motions. They move horizontally at about $10 \mathrm{~km} \mathrm{~s}^{-1}$ and then move downwards so that individual blobs attain speeds of $35 \mathrm{~km} \mathrm{~s}^{-1}$. The downwards acceleration of the blobs is $0.015 \mathrm{~km} \mathrm{~s}^{-2}$ or less, significantly below the gravitational acceleration of $0.27 \mathrm{~km} \mathrm{~s}^{-2}$ (Chae et al. 2008). Also observed are turbulent looking dark features moving upwards from the bottom edge of the hedgerow (Fig. 5). These plumes have maximum initial speeds in the plane of the sky of $20-30 \mathrm{~km} \mathrm{~s}^{-1}$ and decelerate as they rise (Berger et al. 2010).

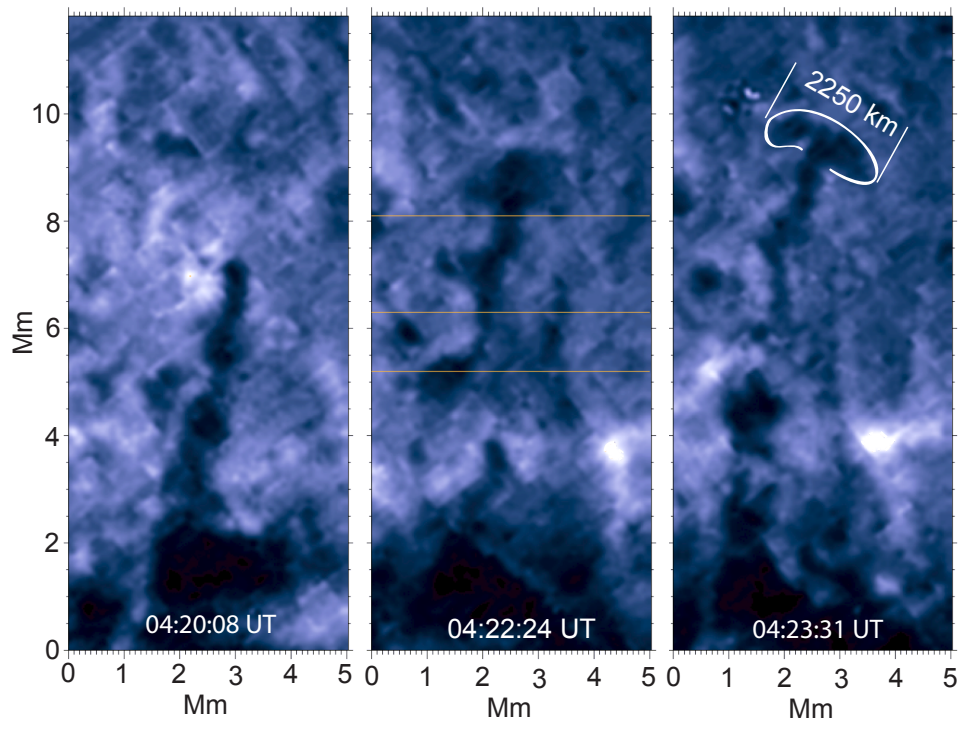

Fig. 5.- Dark upward moving feature with a "mushroom cap" head observed on the limb with Hinode/SOT in the Ca II H (3968 A) line, ascending with a mean velocity of $22.9 \mathrm{~km} \mathrm{~s}^{-1}$ in the plane of the sky (Berger et al. 2008). 
A number of possible explanations for the sub-gravitational acceleration in hedgerows have been suggested. This phenomenon is also observed in coronal cloud prominences and coronal rain (see $\S 1.2 .3$ ), although it is not clear if the same phenomena is at work. Possible mechanisms include density enhancements in vertical magnetic fields (Mackay \& Galsgaard 2001), Lorentz forces associated with small scale horizontal or tangled fields (Low \& Petrie 2005; van Ballegooijen \& Cranmer 2010), and pressure from waves transverse to the field (Pécseli \& Engvold 2000; Antolin \& Verwichte 2011).

Doppler data indicate that the apparently vertical structures in hedgerows also have a velocity component along the line of sight (Schmieder et al. 2010). This may be relevant to both the sub-gravitational acceleration and the question of how these features on the limb relate to those seen on the disk. Contrary to the rather turbulent appearance of hedgerows observed from the side, $\mathrm{H} \alpha$ observations from above show relatively direct flows along straight thread-like structures with inclined up- and down-flows in the barbs. It is not clear how these two points of view are to be reconciled.

\subsubsection{Flows in Active Region Prominences}

One of the signatures of active region prominences is their high level of activity, which includes easily seen flows along the prominence axis. A number of studies report the formation of active region prominences via abrupt jet-like flows, often associated with
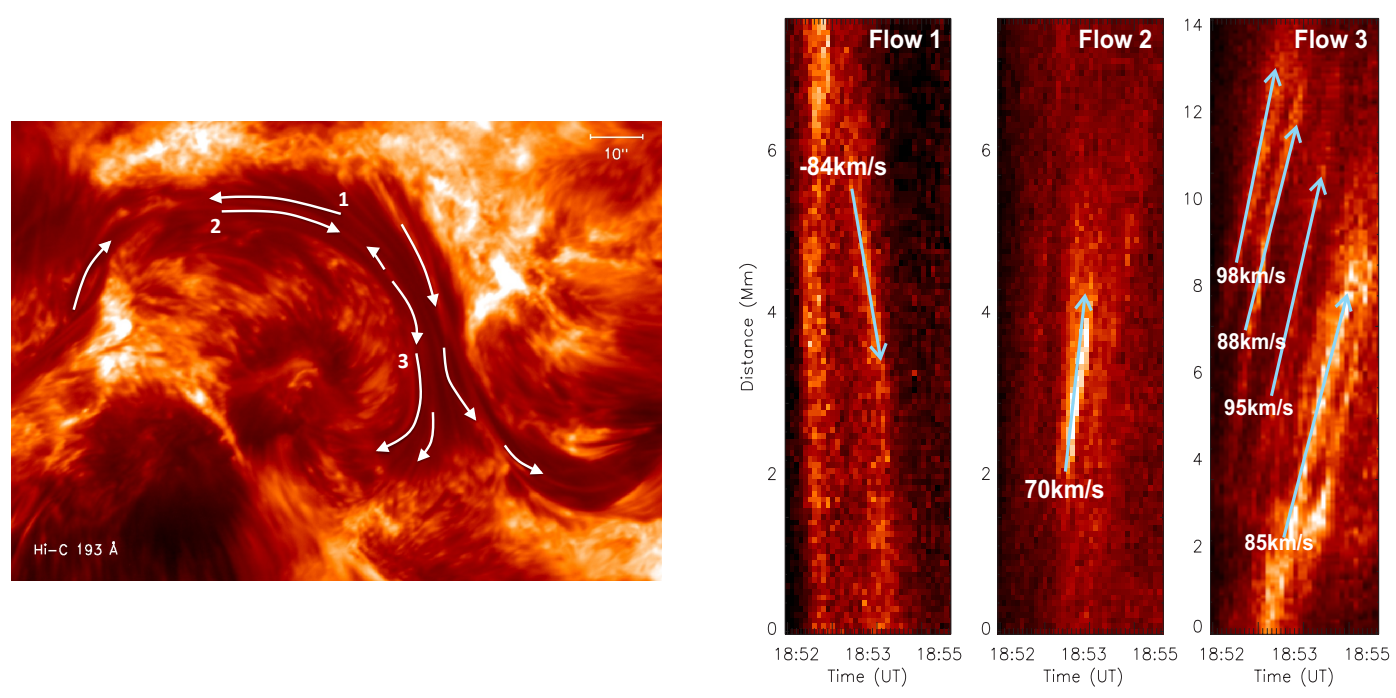

Fig. 6.- Flows observed in an active region filament with the rocket-borne High-resolution Coronal Imager (Hi-C) instrument in a $193 \AA$ band (Alexander et al. 2013). Flows were observed in both directions along the spine with velocities in the plane of the sky between 70 and $100 \mathrm{~km} \mathrm{~s}^{-1}$. 
observed activity in the magnetic field. Velocities in forming active region filaments can be as high as $250 \mathrm{~km} \mathrm{~s}^{-1}$ (Chae 2003). Even once formed the regions show flows along the spine, which include counter-streaming motions in which there are multiple flows in the spine moving in opposite directions. Fig. 6 shows motions of almost $100 \mathrm{~km} \mathrm{~s}^{-1}$ in an active region filament observed in the $193 \AA$ band (Alexander et al. 2013). In addition to these flows observed in cool prominence plasma, active regions filaments are also associated with coronal temperature jet-like features directed parallel to or spiraling around the cooler filament material.

\subsubsection{Coronal Cloud Prominences and Coronal Rain}

Coronal cloud prominences are characterized by drainage along curved trajectories. One study from the 1970's found acceleration in some coronal cloud prominence flows to be consistent with that expected from gravitation (Engvold 1976). More recent observations report accelerations significantly less than gravitational acceleration (Stenborg et al. 2008; Liu et al. 2012b). As discussed in \$1.2.1, a number of theories have been put forward to explain such sub-gravitational acceleration,

The draining of chromospheric temperature material from the corona is know as "coronal rain." Coronal rain is also commonly observed in unstable loops above active regions. Combined EUV and visible observations of such loops show that the phenomena is due to hot, coronal temperature loops cooling, resulting in the raining down of condensing material (Schrijver 2001). This may be due to the sort of evaporation-condensation process hypothesized for prominences (Karpen 2015) but applied to arched field lines instead of the horizontal or dipped field lines associated with prominence inversion lines. As in coronal cloud prominences, the observed acceleration is less than that expected from gravity. Average speeds are about $70 \mathrm{~km} \mathrm{~s}^{-1}$ (Antolin \& Rouppe van der Voort 2012).

\subsubsection{Flows Observed in the PCTR and Corona}

In many ways, observations of the prominence corona transition region (PCTR) and corona in and around prominences are quite limited compared to $\mathrm{H} \alpha$ observations since they rarely reach the combination of high temporal, spatial, and sometimes spectral resolutions available in $\mathrm{H} \alpha$ (although IRIS should offer an improvement on this for some transition region lines). However, the insight they provide with regard to temperature makes them important to our understanding of basic prominence properties.

One key question is how hotter temperature plasma is moving relative to the cool material forming the prominence core. Some observations do show emissions formed at different transition region temperatures from what appear to be the same moving source, 
especially below about $2.5 \times 10^{5} \mathrm{~K}$ (Wiik et al. 1993; Kucera et al. 2003; Kucera \& Landi 2006, 2008), suggesting a cool core with a PCTR accompanying it. Other studies, especially Doppler and related modeling, have suggested that there may be different threads formed at different temperatures with a range of velocities de Boer et al. 1998; Cirigliano et al. 2004).

As described above, EUV observations of transition region temperature material in prominences have shown faster moving plasma than what is considered normal in $\mathrm{H} \alpha$. This may be because they highlight portions of the prominences not observed in $\mathrm{H} \alpha$. However, even observations of absorption near $195 \AA$, which should reflect the same plasma as $\mathrm{H} \alpha$, report quite fast motions (Panasenco et al. 2014). This may be a selection effect resulting from the relatively low spatial resolution of most EUV imagers as compared to the highest resolution $\mathrm{H} \alpha$ telescopes.

The higher temperature transition region lines and even corona lines highlight the extent to which the prominence is part of a larger magnetic structure. In active prominences bright jets of coronal temperature material spiral around prominence spines. Even quiescent prominences viewed in EUV at the limb along direction of the prominence spine reveal flows along apparently spiral tracks inside prominence cavity, while the cool dense prominence material collects along the bottom. Flows in cavities associated with prominences are discussed in detail in Chapter 13 (Gibson 2015).

\subsubsection{Flows on Time Scales of Days}

Prominences evolve on different time scales. For instance, they are been measured to rise and expand with time, especially in the days before eruption (Liu et al. 2012a). Such changes are presumably due to evolution of the magnetic field in which the prominence is embedded (Gibson 2015).

Another sort of slow evolution has been reported in stable filaments. Kilper et al. (Kilper et al. 2009) report that such filaments show a decrease in ratio of He I $10830 \AA$ to $\mathrm{H} \alpha$ emission as a function of height over time, although this variation disappears in more active or erupting filaments. They interpret this as sign of cross field diffusion which would be expected to be much faster for the heavier helium atoms than for hydrogen (Gilbert et al. 2007).

\section{Prominence Mass}

Prominence mass is an important quantity in our understanding of prominence plasma - a basic physical quantity to be accounted for by models of prominence formation and also eruption. Here we will discuss methods used for measuring prominence mass and summarize 
some of the results.

\subsection{Continuum Absorption}

The most frequently used method for measuring prominence mass is via measurements of continuum absorption. An example of a prominence seen in absorption in EUV is shown in Fig. 7. This is absorption due to the photoionization of neutral hydrogen and neutral and once ionized helium, chiefly Lyman-absorption from the ground state, and is proportional to $\exp (-\sigma N)$ where $N$ is the column number of absorbing atoms or ions and $\sigma$ is the absorption cross section. As shown in Fig. 8, the absorption cross section for each of these species has an upper limit in wavelength, $912 \AA$ for $\mathrm{H}^{0}, 504 \AA$ for $\mathrm{He}^{0}$, and $228 \AA \mathrm{He}^{+}$ below which it decreases. There are also auto-ionization resonances in the neutral helium cross section. Formulations for the individual cross sections can be found in a number of sources (Karzas \& Latter 1961; Fernley et al. 1987; Rumph et al. 1994; Keady \& Kilcrease 2000 ).

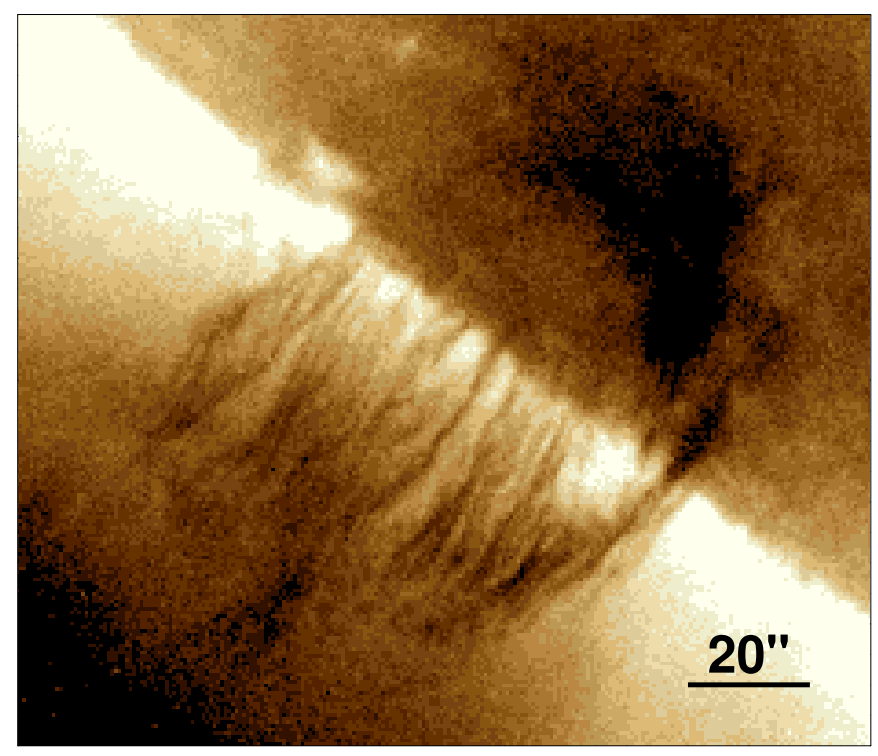

Fig. 7.- Prominence observed in absorption in the $193 \AA$ band of SDO/AIA on 2010 August 11 at 20:00 UT. 
The total cross section, $\sigma$ is

$$
\begin{aligned}
\sigma & =N_{H^{0}} \sigma_{H}+N_{H e^{0}} \sigma_{H e^{0}}+N_{H e^{+}} \sigma_{H e} \\
& =\epsilon_{H}\left(1-x_{H}\right) \sigma_{H}+\left(1-\epsilon_{H}\right)\left(1-x_{H e^{+}}-x_{H e^{+2}}\right) \sigma_{H e^{0}}+\epsilon_{H e} x_{H e^{+}} \sigma_{H e} .
\end{aligned}
$$

Here $\epsilon_{H}$ and $\epsilon_{H e}$ are the fractional abundances of hydrogen and helium by number $\left(\epsilon_{H}+\epsilon_{H e} \approx 1\right)$, and $x_{H}, x_{H e^{+}}$and $x_{H e^{+2}}$ are the ionization fractions of $\mathrm{H}$ and He in the absorbing region. We usually assume that all helium is neutral or singly ionized in the region of interest, so that $x_{H e^{+2}}=0$.

Possible ranges for these quantities would be: $0.1 \gtrsim x_{H} \gtrsim 0.5,0.005 \gtrsim x_{H e} \gtrsim 0.14$, and $0.85 \gtrsim \epsilon_{H} \gtrsim 0.95$ (Gilbert et al. 2005).

Continuum absorption in prominences was first noted in Skylab data. Orrall \& Schmahl (Orrall \& Schmahl 1976, 1980), used absorption measurements to determine column densities of neutral hydrogen. Similar measurements were carried out with SOHO (Kucera et al. 1998; Penn 2000) and TRACE (Golub et al. 1999), and have been performed using a number of different instruments and combinations thereof. Typical $\mathrm{H}$ I column densities are on the order $10^{18}-10^{19} \mathrm{~cm}^{-2}$, but larger and smaller values have been reported. Clearly, the exact values will depend on the particular feature analyzed and its orientation. Gilbert et al. (Gilbert et al. 2005, 2006) first used absorption-based column density measurements to estimate the mass for entire prominences. They found total prominence mass values in the range $8 \times 10^{13}-2 \times 10^{15} \mathrm{~g}$.

The basic geometry of the problem is as shown in Fig. 9. In this formulation $I_{0}$ is the intensity that would be seen by the observer if there were no prominence, while $I_{1}$ is the intensity in the portion of the sky containing the prominence material. In areas not including the prominence in the line of sight these are equal. $I_{0}$ in the region containing the prominence is determined by either interpolating spatially using adjacent areas without prominence material or interpolating temporally if the prominence material is moving across the field of view.

In each case the observed intensity is comprised of three parts along the line of sight: the intensity in the foreground, $I_{f}$, the background, $I_{b}$ and from the region of the prominence itself, $I_{p}$.

$$
\begin{gathered}
I_{0}=I_{b}+I_{0 p}+I_{f}, \\
I_{1}=\alpha I_{b}+I_{1 p}+I_{f} .
\end{gathered}
$$

where the absorption is represented by the extinction factor,

$$
\alpha=\exp \left(-\int_{0}^{l} n \sigma d s\right),
$$

in which the product of the number density of particles and the absorption cross section in the source is integrated over the depth of the prominence along the line of sight, $l$. 


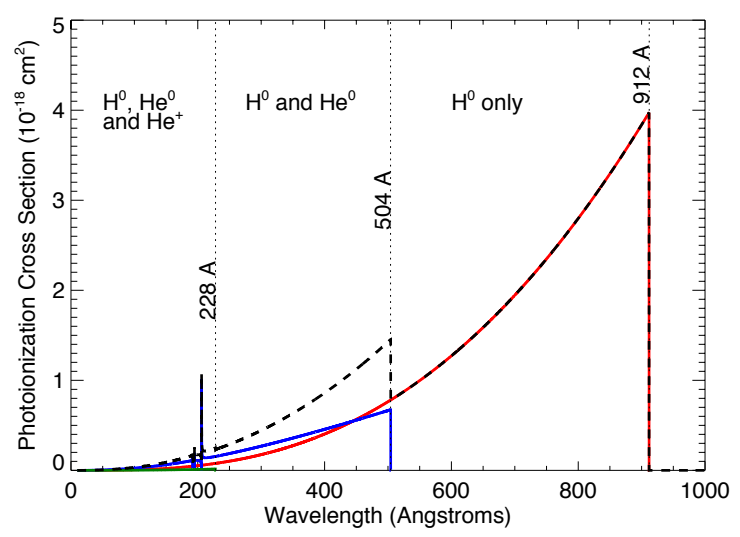

Fig. 8.- Photoionization cross-section per atom/ion in a prominence of assumed hydrogen fraction 0.9, hydrogen fractional ionization 0.3 and helium ionization fraction 0.1. Neutral hydrogen (Karzas \& Latter 1961) (red) contributes below $912 \AA, \mathrm{He}^{0}$ (Fernley et al. 1987) (blue) below $504 \AA$ and $\mathrm{He}^{+}$(Karzas \& Latter 1961) (green) below $228 \AA$. The He I curve shows the locations of the helium auto-ionization features. The total average is shown by the dashed line. Code courtesy V. Andretta.
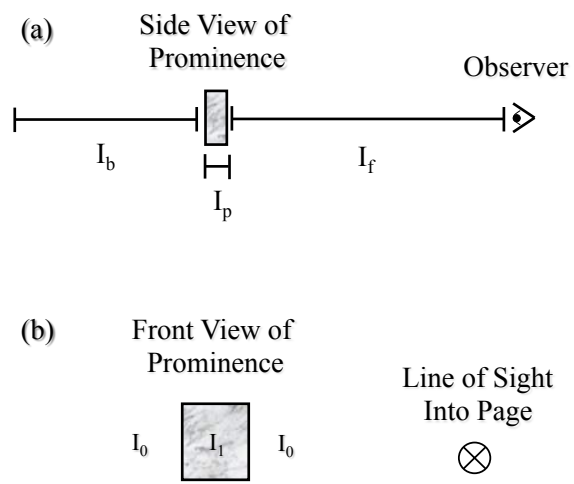

Fig. 9.- Schematics showing the a prominence observed from (a) the side and (b) the front (Gilbert et al. 2005). (a) $I_{b}$ and $I_{f}$ represent the emission in the background and foreground of the prominence while $I_{p}$ is represents the coronal-temperature radiation from the region of the prominence. (b) $I_{0}$ represents the observed intensity in areas of the sky without the prominence while $I_{1}$ represents the intensity with the prominence. 
The total mass is then

$$
M=\left(4\left(1-\epsilon_{H}\right)+\epsilon_{H}\right) m_{H} \iint-\frac{\ln \alpha}{\sigma} d a,
$$

where $m_{H}$ is the mass of hydrogen and $a$ is the area associated with the prominence absorption.

Thus there are at least five unknowns connected to the geometry alone. There are additional ones associated with the abundance and ionization parameters (Eq. 8) and other issues like unresolved structure and portions of the prominence not detected in absorption. Researchers using continuum absorption techniques to calculate column densities and prominence mass have approached these problems with a variety of assumptions and combinations of data.

Geometry For a prominence sufficiently in front of the plane of the sky (erupting from near disk center, for instance) one might assume $I_{f}<<I_{b}$ and neglect the foreground radiation all together. It has also been suggested that for a prominence on the limb one might assume $I_{f} \approx I_{b}$, although the corona is highly non-uniform, so such an assumption could be problematic.

Gilbert et al (Gilbert et al. 2005) developed a technique for estimating $I_{f}$ and $I_{b}$ by comparing the prominence emission against adjacent regions in which the background but not the foreground emission is presumed to be varying (at the solar limb, for instance).

The Extended EUV Filament Some EUV observations reveal a more extended filament structure than that often considered in the $\mathrm{H} \alpha$ line. $\mathrm{H} \alpha$ is similar in optical depth to the continuum absorption near about $195 \AA$ (Heinzel et al. 2008). Thus, as can be seen from Fig. 8, observations of filaments in wavelengths that are significantly greater than $195 \AA$ but still less than the Lyman cut-off at 912 would be expected to show more tenuous portions of the prominence. It is indeed the case that EUV observations of filament channels, for instance in $\mathrm{Mg}$ X $625 \AA$, show substantially wider filaments than those observed in $\mathrm{H} \alpha$ (Heinzel et al. 2003; Schwartz et al. 2004). Extended filament structure is also observed in hydrogen Lyman- $\alpha$ observations of prominences (Vial et al. 2012; Schwartz et al. 2012) and, as mentioned earlier in this chapter, prominences appear much larger in He II $304 \AA$ than in $\mathrm{H} \alpha$. Fig. 10 shows a suggested geometry for the extended filament based on H-Ly $\alpha$ observations. It is not clear in such lines the extent to which we are observing more diffuse cool material or starting to observe the PCTR. It has been estimated that the extra mass due to the filament extension may be equal to $50-100 \%$ the mass derived from the $\mathrm{H} \alpha$ emitting prominence (Aulanier \& Schmieder 2002; Heinzel et al. 2003).

Emissivity Blocking Emissivity Blocking (at one time referred to as volume blocking) is a reduction in the observed intensity in the corona due to the fact that cool structures do not emit radiation in lines formed at coronal temperatures (Anzer \& Heinzel 2005; Heinzel et al. 2008). This applies both to the situation where the prominence is observable via 


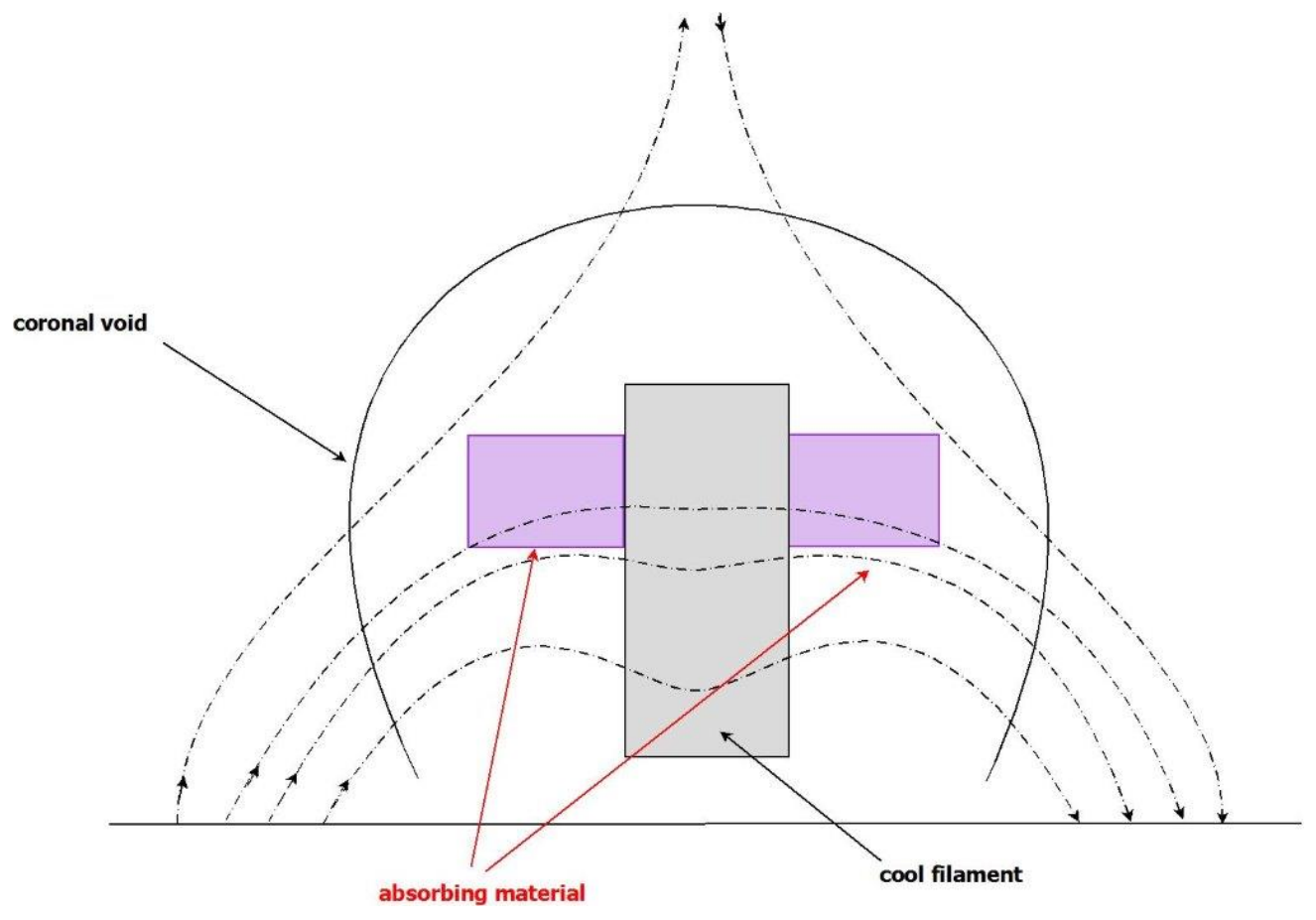

Fig. 10. - Cartoon based on H-Ly $\alpha$ observations (Vial et al. 2012) showing the location of the extended EUV filament in a filament channel viewed along the inversion line. The central "cool filament" area shows the location of the prominence as would be seen in $\mathrm{H} \alpha$. The "absorbing material" shows additional areas with material detected in Ly $\alpha$. Also shown is the outline of the coronal cavity ("coronal void") and magnetic field lines (dashed lines). 
absorption, and also to coronal line observations at wavelengths not effected by continuum absorption or where such absorption is relatively weak. In these later cases there may be darkening due to emissivity blocking alone. Measurement of emissivity blocking can be performed with a combination of data taken at wavelengths that do not exhibit continuum absorption and ones that do. These can be combined to determine the amount of darkening which occurs simply because of a lack of emission, allowing for a accurate accounting for the $I_{0 p}$ and $I_{1 p}$ terms in Eqs. 4 and 5 .

Substructure Unresolved sub-structure can also affect masses deduced from continuum absorption (Orrall \& Schmahl 1980; Kucera et al. 1998, 2014). One way to account for such sub-structure is through a filling factor describing the fraction of the observing element that is filled with material. This quantity, $f_{p o s}$, is an area filling factor and is distinct from the volume filling factor. Including it changes Eq. 6 to:

$$
\alpha=f_{p o s} \exp \left(-\int_{0}^{l} n \sigma d s\right)+\left(1-f_{p o s}\right)
$$

If $f_{\text {pos }}$ is low then the amount of material needed to account for a measured amount of absorption, $\alpha$, will be higher than that needed if there is no unresolved substructure.

Prominence Emission It is also often assumed that $I_{1 p}=0$, i.e., that the emission from the prominence itself is negligible. However, this may not be the case in some wave bands containing contributions from the PCTR. In general when using continuum absorption it is best to select lines or wavebands in which there is no extra emission from the prominence itself and substantial background emission.

\section{Other Uses for Continuum Absorption Measurements in Prominences} Uncertainties in ionization fraction and helium abundance present difficulties for mass determinations using Lyman-continuum absorption. The converse of that is that this absorption has potential for measurements of abundances and temperature. Gilbert et al. (Gilbert et al. 2011) attempted to calculate the neutral $\mathrm{H} / \mathrm{He}$ ratio by using $\mathrm{SOHO}$ CDS data above and below the $504 \AA$ for neutral helium absorption. The attempt was foiled by optically thick absorption in one of the lines used ( $\mathrm{Mg} \mathrm{X}$ at $625 \AA$ ), which is another thing to be careful of when analyzing absorption. However, the measurement may be possible with a more sensitive instrument able to detect fainter coronal lines and and fainter features. Landi \& Reale (Landi \& Reale 2013) use the dependence of the continuum absorption on ionization fraction and thus temperature to estimate the temperature in an eruptive prominence.

\subsection{Cloud Modeling}

Mass can be determined with spectral lines by comparing observations with the results of non-LTE magneto-hydrostatic models (Mein et al. 1996; Heinzel et al. 1999; 
Heinzel 2015). By measuring or making assumptions about temperature, bulk velocity and turbulent velocity it is possible use a grid of model results combined with measurements of the filament dimensions to calculate the filament mass. As with the continuum absorption method, uncertainties in ionization fractions result in significant uncertainties in the mass values. Masses calculated in this way give values in the range $2-6 \times 10^{15} \mathrm{~g}$, on the order of values obtained using calculations based on continuum absorption (Koutchmy et al. 2008; Grechnev et al. 2014).

\subsection{White Light Measurements}

Erupting prominences contribute to the mass calculated for CMEs done using white light coronagraph observations. If the emission observed is purely due to Thompson scattering off electrons then mass is proportional to the electron density with an angle dependence emphasizing the contribution in the plane of the sky. Inversions of the white light intensity to calculate the CME mass have been performed since the 1970s (Stewart et al. 1974).

To calculate CME mass the white light associated with the CME is determined by subtracting off a pre-event image. The mass is then (Vourlidas et al. 2010):

$$
M_{C M E}=I_{C M E} C_{e} C_{\text {plasma }},
$$

where $I_{C M E}$ is the excess brightness associated with the CME in units of mean solar brightness and $C_{\text {plasma }}$ is the composition of the CME plasma. If one assumes a composition of $10 \%$ helium $C_{\text {plasma }}$ is $2 \times 10^{-24}$ g electron ${ }^{-1}$. $C_{e}$ is obtained from Thompson scattering theory (Billings 1966; Hundhausen 1993) using the assumption that all electrons lie in the plane of the sky. The mass is determined for each pixel and summed over the area associated with the CME.

It is also usually assumed that the prominence material still present is completely ionized (Vourlidas et al. 2010). If that is not the case it can result in an over estimate of CME mass due to $\mathrm{H} \alpha$ emission in the white light bandpass. Athay and Illing (Athay \& Illing 1986) took into account both white light and $\mathrm{H} \alpha$ to calculate the mass of an erupting prominence, including an analysis of the ionization state of hydrogen, to obtain a mass of $1 \times 10^{16} \mathrm{~g}$.

\section{Some Outstanding Questions Related to Prominence Flows and Mass}

There are numerous questions concerning prominences to which measurements of flows and mass are key. Most of them tie into the basic question of the origins of prominence mass. Clearly we need to bring all available observations of flows and oscillations along with 
measurements of temperature, mass, density, and magnetic field and, of course, modeling to bear on this larger question and the related questions listed below.

- Where and at what temperature does prominence plasma originate? This is an important distinguisher between different theories of the origin of prominence mass. Some observations, especially in active regions, suggest that prominence material originates as relatively cool plasma ejected from the chromosphere (Wang 1999; Schmieder et al. 2004). Other observations suggest that the cool material condenses from hot material (Berger et al. 2012). These differences may be the result of more than one mechanism at work in different situations, for instance between active region vs. quiescent prominences, but this still is not entirely clear.

- In a related question, what is the role of barbs? Are they the chief conduit of material to and from the chromosphere, important to the existence of prominence plasma, or are they only the result of perturbations on the prominence channel magnetic field with no special role in the processes moving material into the prominence?

- The complex flows in hedgerow prominences offer a fascinating challenge, offering us a look into a regime in which apparently different processes dominate than in lower latitude prominences. Further detailed observations of these motions in three dimensions (spectra with high spatial and temporal resolution combined with high quality imaging) and magnetic field information (Orozco Suárez et al. 2014) should help us understand the processes at work.

- How are the plasmas in the prominence and the larger prominence cavity related? Three dimensional morphological modeling using EUV images and coronal magnetic field measurements could help us understand the prominence cavity system.

- What determines the size and structure of prominence flows at different scales? Substructure may reflect the manner in which the material is inserted into the corona (Kucera et al. 2014), or, alternatively, be a result of processes occurring in the prominence itself, including evolution of the magnetic field or wave based variations (Antolin et al. 2014).

The understanding of flows and mass distributions in prominences is central to these and other important questions about the nature of these complicated and mysterious features of the solar atmosphere. 


\section{REFERENCES}

Ahn, K., Chae, J., Cao, W., \& Goode, P. R. 2010, ApJ, 721, 74

Alexander, C. E., Walsh, R. W., Régnier, S., et al. 2013, ApJ, 775, L32

Antolin, P., \& Rouppe van der Voort, L. 2012, ApJ, 745, 152

Antolin, P., \& Verwichte, E. 2011, ApJ, 736, 121

Antolin, P., Yokoyama, T., \& Van Doorsselaere, T. 2014, ApJ, 787, L22

Anzer, U., \& Heinzel, P. 2005, ApJ, 622, 714

Athay, R. G., \& Illing, R. M. E. 1986, J. Geophys. Res., 91, 10961

Aulanier, G., \& Schmieder, B. 2002, A\&A, 386, 1106

Ballester, J. 2015, in Solar Prominences, ed. J.-C. Vial \& O. Engvold (Springer)

Beckers, J. M. 1964, PhD thesis, Sacramento Peak Observatory, Air Force Cambridge Research Laboratories, Mass., USA

Berger, T. E., Liu, W., \& Low, B. C. 2012, ApJ, 758, L37

Berger, T. E., Shine, R. A., Slater, G. L., et al. 2008, ApJ, 676, L89

Berger, T. E., Slater, G., Hurlburt, N., et al. 2010, ApJ, 716, 1288

Billings, D. E. 1966, A Guide to the Solar Corona (New York: Academic Press)

Chae, J. 2003, ApJ, 584, 1084

Chae, J., Ahn, K., Lim, E.-K., Choe, G. S., \& Sakurai, T. 2008, ApJ, 689, L73

Chae, J., \& Sakurai, T. 2008, ApJ, 689, 593

Cirigliano, D., Vial, J.-C., \& Rovira, M. 2004, Sol. Phys., 223, 95

de Boer, C. R., Stellmacher, G., \& Wiehr, E. 1998, A\&A, 334, 280

Engvold, O. 1976, Sol. Phys., 49, 283

-. 2015, in Solar Prominences, ed. J.-C. Vial \& O. Engvold (Springer)

Fernley, J. A., Seaton, M. J., \& Taylor, K. T. 1987, Journal of Physics B Atomic Molecular Physics, 20, 6457

Gibson, S. 2015, in Solar Prominences, ed. J.-C. Vial \& O. Engvold (Springer) 
Gilbert, H., Kilper, G., \& Alexander, D. 2007, ApJ, 671, 978

Gilbert, H., Kilper, G., Alexander, D., \& Kucera, T. 2011, ApJ, 727, 25

Gilbert, H. R., Falco, L. E., Holzer, T. E., \& MacQueen, R. M. 2006, ApJ, 641, 606

Gilbert, H. R., Holzer, T. E., \& MacQueen, R. M. 2005, ApJ, 618, 524

Golub, L., Bookbinder, J., Deluca, E., et al. 1999, Physics of Plasmas, 6, 2205

Gopalswamy, D. 2015, in Solar Prominences, ed. J.-C. Vial \& O. Engvold (Springer)

Grechnev, V. V., Uralov, A. M., Slemzin, V. A., et al. 2014, Sol. Phys., 289, 289

Heinzel, P. 2015, in Solar Prominences, ed. J.-C. Vial \& O. Engvold (Springer)

Heinzel, P., Anzer, U., Schmieder, B., \& Schwartz, P. 2003, in ESA Special Publication, Vol. 535, Solar Variability as an Input to the Earth's Environment, ed. A. Wilson, $447-457$

Heinzel, P., Mein, N., \& Mein, P. 1999, A\&A, 346, 322

Heinzel, P., Schmieder, B., Fárník, F., et al. 2008, ApJ, 686, 1383

Hundhausen, A. J. 1993, J. Geophys. Res., 98, 13177

Karpen, J. 2015, in Solar Prominences, ed. J.-C. Vial \& O. Engvold (Springer)

Karzas, W. J., \& Latter, R. 1961, ApJS, 6, 167

Keady, J. J., \& Kilcrease, D. P. 2000, in Allens Astrophysical Quantities, ed. A. N. Cox (New York: AIP), 95-120

Kilper, G., Gilbert, H., \& Alexander, D. 2009, ApJ, 704, 522

Koutchmy, S., Slemzin, V., Filippov, B., et al. 2008, A\&A, 483, 599

Kucera, T. A., Andretta, V., \& Poland, A. I. 1998, Sol. Phys., 183, 107

Kucera, T. A., Gilbert, H. R., \& Karpen, J. T. 2014, ApJ, in press

Kucera, T. A., \& Landi, E. 2006, ApJ, 645, 1525

-. 2008, ApJ, 673, 611

Kucera, T. A., Tovar, M., \& de Pontieu, B. 2003, Sol. Phys., 212, 81

Landi, E., \& Reale, F. 2013, ApJ, 772, 71 
Leese, J. A., Novak, C. S., \& Taylor, V. R. 1970, Pattern Recognition, 2, 279280

Lin, Y., Engvold, O. R., \& Wiik, J. E. 2003, Sol. Phys., 216, 109

Liu, K., Wang, Y., Shen, C., \& Wang, S. 2012a, ApJ, 744, 168

Liu, W., Berger, T. E., \& Low, B. C. 2012b, ApJ, 745, L21

Low, B. C., \& Petrie, G. J. D. 2005, ApJ, 626, 551

Mackay, D. H., \& Galsgaard, K. 2001, Sol. Phys., 198, 289

Mein, N., Mein, P., Heinzel, P., et al. 1996, A\&A, 309, 275

November, L. J., \& Simon, G. W. 1988, ApJ, 333, 427

Orozco Suárez, D., Asensio Ramos, A., \& Trujillo Bueno, J. 2012, ApJ, 761, L25

Orozco Suárez, D., Díaz, A. J., Asensio Ramos, A., \& Trujillo Bueno, J. 2014, ApJ, 785, L10

Orrall, F. Q., \& Schmahl, E. J. 1976, Sol. Phys., 50, 365

—. 1980, ApJ, 240, 908

Panasenco, O., Martin, S. F., \& Velli, M. 2014, Sol. Phys., 289, 603

Pécseli, H., \& Engvold, O. 2000, Sol. Phys., 194, 73

Penn, M. J. 2000, Sol. Phys., 197, 313

Rumph, T., Bowyer, S., \& Vennes, S. 1994, AJ, 107, 2108

Schmieder, B., Chandra, R., Berlicki, A., \& Mein, P. 2010, A\&A, 514, A68

Schmieder, B., Mein, N., Deng, Y., et al. 2004, Sol. Phys., 223, 119

Schrijver, C. J. 2001, Sol. Phys., 198, 325

Schuck, P. W. 2006, ApJ, 646, 1358

Schwartz, P., Heinzel, P., Anzer, U., \& Schmieder, B. 2004, A\&A, 421, 323

Schwartz, P., Schmieder, B., Heinzel, P., \& Kotrč, P. 2012, Sol. Phys., 281, 707

Stenborg, G., Vourlidas, A., \& Howard, R. A. 2008, ApJ, 674, 1201

Stewart, R. T., McCabe, M. K., Koomen, M. J., Hansen, R. T., \& Dulk, G. A. 1974, Sol. Phys., 36, 203 
Tziotziou, K. 2007, in Astronomical Society of the Pacific Conference Series, Vol. 368, The Physics of Chromospheric Plasmas, ed. P. Heinzel, I. Dorotovič, \& R. J. Rutten (San Francisco: Astronomical Society of the Pacific), 217

van Ballegooijen, A. A., \& Cranmer, S. R. 2010, ApJ, 711, 164

Vial, J.-C., Olivier, K., Philippon, A. A., Vourlidas, A., \& Yurchyshyn, V. 2012, A\&A, 541, A108

Vourlidas, A., Howard, R. A., Esfandiari, E., et al. 2010, ApJ, 722, 1522

Wang, Y.-M. 1999, ApJ, 520, L71

Webb, D. 2015, in Solar Prominences, ed. J.-C. Vial \& O. Engvold (Springer)

Wiik, J. E., Dere, K., \& Schmieder, B. 1993, A\&A, 273, 267

Zirker, J. B., Engvold, O., \& Martin, S. F. 1998, Nature, 396, 440 